\title{
FINE STRUCTURE DISCUSSION OF PARITY-NONCONSERVING NEUTRON SCATTERING AT EPITHERMAL ENERGIES *
}

\author{
M. S. Hussein ${ }^{\dagger}$, A. K. Kerman and C-Y Lin ${ }^{\ddagger}$ \\ Center for Theoretical Physics \\ Laboratory for Nuclear Science \\ and Department of Physics \\ Massachussets Institute of Technology \\ Cambridge, Massachusetts 02139 U.S.A.
}

\begin{abstract}
The large magnitude and the sign correlation effect in the parity non-conserving resonant scattering of epithermal neutrons from ${ }^{232} \mathrm{Th}$ is discussed in terms of a non-collective $2 p-1 h$ local doorway model. General conclusions are drawn as to the probability of finding large parity violation effects in other regions of the periodic table.
\end{abstract}

PACS numbers: 25.40.Ny, 11.30.Er, 24.10.Ht, 24.80.Dc

CTP\# 2296

September 08, 1994

* This work is supported in part by funds provided by the U. S. Department of Energy (D.O.E.) under cooperative agreement \#DE-FC02-94ER40818.

$\dagger$ Permanent address: Instituto de Física, Universidade de São Paulo, C. P. 20516, São Paulo S. P. Brazil. Supported partly by FAPESP (Brazil)

$\ddagger$ Supported by Conselho de Desenvolvimento Científico e Tecnológico(CNPq) 
The discovery of sign correlation in parity non-conserving (PNC) epithermal neutroninduced compound-nucleus reactions involving the heavy nucleus ${ }^{232} \mathrm{Th}$ has prompted intensive theoretical discussion concerning their origin. The great interest in the TRIPLE data [1]stems from the fact that the statistical theory (ST) of these reactions, although it predicts the possibility of large PNC at individual resonances as the data also show, rules out any sign correlations in the longitudinal asymmetry, contrary to what the data show.

The quantity usually analysed is the longitudinal asymmetry. At a given $p 1 / 2$ resonance in the compound nucleus

$$
P=\frac{\sigma_{R}^{(+)}-\sigma_{R}^{(-)}}{\sigma_{R}^{(+)}+\sigma_{R}^{(-)}}
$$

where $\sigma_{R}^{( \pm)}$is the resonance neutron cross-section for \pm helicities at a given $p 1 / 2$ resonance. We now use the usual formula for the total cross-section

$$
\sigma_{T}^{( \pm)}=\frac{4 \pi}{k} \operatorname{Im} f^{( \pm)}(0)=\sigma_{0}^{( \pm)}+\sigma_{R}^{( \pm)}
$$

with

$$
f^{( \pm)}(0)=f_{0}^{ \pm}-\frac{1}{2 k} \sum_{q} \frac{\gamma_{q}\left(\gamma_{q} \pm \gamma_{q}^{W}\right)}{E-E_{q}+i \Gamma_{q} / 2}
$$

where $\sigma_{0}^{ \pm}$is a background non-resonant piece related to $f_{0}^{( \pm)}$, the latter being roughly equal to $-a^{( \pm)}$with $a^{( \pm)}$being the scattering length, $k$ is the asymptotic wave number, $E_{q}-i \Gamma_{q} / 2$ is the complex energy of the $q^{\text {th }}$ resonance $(p 1 / 2)$ in the compound nucleus, $\Gamma_{q}$ is the total width (including $\gamma$-decay), $\gamma_{q}$ is the strong component of the neutron decay amplitude and $\gamma_{q}^{W}$ is the "weak" component of the neutron decay amplitude arising from coupling of the $s 1 / 2$ and $p 1 / 2$ channels and measures the PNC strength. Thus one has

$$
P=\frac{\Delta \sigma_{R}}{2 \sigma_{R}}=\frac{\gamma^{W}}{\gamma}
$$

for each resonance and the average $\langle P\rangle_{q}=\left\langle\gamma^{W} / \gamma\right\rangle_{q}$ was found to be about $0.08 \pm 0.06$ (cf Ref.[1] ). Theoretical attempts to explain the large value of $\langle P\rangle_{q}$ have been made using different single particle mechanisms [2] [3] [4] [5]. So far no explanation has been put forth which does not require on unrealistically large parity violating matrix element, of about $100 \mathrm{eV}$ $[6]$. 
We discuss in what follows the fine structure aspect of the data [7]. A very natural mechanism that could account for this sign correlation is to assume that the compound nuclear process occurs through a single dominantly $p$-wave local doorway which contains a small parity violation. For simplicity we start with this extreme hypothesis. Below we will consider the more general case including more doorways. This doorway is a relatively simple but statistical combination of two particle-one hole $(2 p-1 h)$ states in ${ }^{233} \mathrm{Th}$. In passing we note that it is the dominance of local doorways which may give rise to intermediate structure in the energy dependence of nuclear cross-sections and their statistical nature which gives rise to fluctuations in strength functions from nucleus to nucleus over and above the general optical model trend [8] [9]. We stress that our local doorway states are statistical in nature, in contrast to the collective $O^{-}$doorway (giant monopole) states considered by Auerbach[3][4]as responsible for the sign correlation. We assume that parity violation occurs through the coupling of our $p$-wave doorway to an $s$-wave doorway, located nearby. Then

$$
\gamma_{q}=C_{q D} \gamma_{D p} \quad \gamma_{q}^{W}=C_{q D} \gamma_{D s}^{W}
$$

where $C_{q D}$ are taken to be random. Taking $D$ to be in the vicinity of the compound resonances in question, we obtain with the aid of perturbation theory

$$
P=\frac{\gamma_{D s}^{W}}{\gamma_{D p}} \simeq \frac{\bar{M}^{W}}{\Delta E} \frac{\gamma_{D s}}{\gamma_{D p}}
$$

which represents the average value of $P$, being independent of $q$. In Eq.(6) $\bar{M}^{W}$ is a characteristic "weak" matrix element between $p$ - and $s$-doorways and $\Delta E$ is the corresponding characteristic energy distance between these doorway states given by, e.g.,

$$
\Delta E \simeq\left|\left(E_{D_{2}}-i \frac{\Gamma_{D_{2}}}{2}\right)-\left(E_{D_{1}}+i \frac{\Gamma_{D_{1}}}{2}\right)\right|=\left[\left(E_{D_{2}}-E_{D_{1}}\right)^{2}+\frac{\left(\Gamma_{D_{1}}+\Gamma_{D_{2}}\right)^{2}}{4}\right]^{1 / 2}
$$

In order to estimate the size of (6) we take the smooth energy dependence out of the partial width amplitude $\gamma_{D}$ in the usual fashion, i.e. we define $\gamma_{D s} / \gamma_{D p}=(1 / k R)\left(\gamma_{D s}^{(0)} / \gamma_{D p}^{(0)}\right)$, where $\gamma^{(0)}$ are the reduced widths. Thus

$$
\langle P\rangle=\frac{\bar{M}^{W}}{\Delta E} \frac{\gamma_{D s}^{(0)}}{\gamma_{D p}^{(0)}} \frac{1}{k R},
$$


which will have a definite sign for a given nucleus as seen in the data.

Properties of simple two particle-one hole states can be deduced from the exiton model, usually employed in pre-equilibrium studies[9]. The density of $2 p-1 h$ doorway states coupled to total angular momentum $J$ at an excitation energy $E^{*}$ in the compound nucleus is given by $[9]$

$$
\rho_{2 p-1 h}\left(E^{*}, J\right)=\frac{g^{3} E^{*^{2}}}{4} \frac{(2 J+1) \exp \left[-(J+1 / 2) / 3 \sigma^{2}\right]}{(27 \pi)^{1 / 2} \sigma^{3}}
$$

where $g$ is the average spacing of single particle levels, and $\sigma$ is the spin cut-off parameter. For the deformed nucleus ${ }^{233} \mathrm{Th}, g \sim 10 \mathrm{MeV}^{-1}, \sigma \simeq 4.0$, and taking $E^{*}=6 \mathrm{MeV}, \mathrm{J}=1 / 2$, we find $\rho_{2 p-1 h} \simeq 34 M e V^{-1}$. Thus the average spacing, $D_{2 p-1 h}=\left(\rho_{2 p-1 h}\right)^{-1} \simeq 30 \mathrm{KeV}$. For a simple local doorway to dominate the $2 p-1 h$ doorways must not be overlapping. Thus we take $\Gamma \simeq D \sim 30 \mathrm{KeV}$. Then $\Delta E$ of Eq.(7)becomes roughly $\Delta E \sim \sqrt{2} \Gamma_{D} \sim 50 \mathrm{KeV}$.

Taking for $\bar{M}^{W} \sim 1.0 \mathrm{eV}[10]$ and $k R \sim 10^{-3}$ for $E_{n} \sim 1 \mathrm{eV}$, we find that the data $(\langle P\rangle=0.08)$ require

$$
\left|\frac{\gamma_{D s}^{(0)}}{\gamma_{D p}^{(0)}}\right| \simeq 4
$$

which seems to contradict the fact that p-waves are resonant while s-waves are off resonance. This requires an enhancement which may come about if the particular statistical doorway involved in $\bar{M}^{W}$ couples strongly to $s$ and less so to $p$ or if the particular matrix element $\bar{M}_{W}$ is larger than average. This will be a statistical phenomenon associated with random properties of the local doorways.

The fluctuation part of $P$ can also be analysed within the local doorway model. If we consider "nearby" local doorways which for simplicity we collectively call $D^{\prime}$, then

$$
\gamma_{q}=C_{q D} \gamma_{D p}+C_{q D^{\prime}} \gamma_{D^{\prime} p} \quad \gamma_{q}^{W}=C_{q D} \gamma_{D s}^{W}+C_{q D^{\prime}} \gamma_{D^{\prime} s}^{W}
$$

where

$$
C_{q D^{\prime}}<C_{q D}
$$

on average. Then

$$
P \simeq \frac{\gamma_{D s}^{W}}{\gamma_{D p}}+\frac{C_{q D^{\prime}}}{C_{q D}}\left[\frac{\gamma_{D^{\prime} s}^{W}}{\gamma_{D s}^{W}}-\frac{\gamma_{D^{\prime} p}}{\gamma_{D p}}\right]
$$


The variance of $P$ is then given by

$$
v \equiv \sqrt{\left\langle P^{2}\right\rangle-\langle P\rangle^{2}} \simeq \sqrt{\left\langle\left|\frac{C_{q D^{\prime}}}{C_{q D}}\right|^{2}\right\rangle}\left|\frac{\gamma_{D^{\prime} s}^{W}}{\gamma_{D s}^{W}}-\frac{\gamma_{D^{\prime} p}}{\gamma_{D p}}\right| .
$$

From Ref.[1] we find $v$ to be about unity. Because of (11)

$$
\left|\frac{\gamma_{D^{\prime} s}^{W}}{\gamma_{D s}^{W}}-\frac{\gamma_{D^{\prime} p}}{\gamma_{D p}}\right|>1
$$

which is entirely reasonable. Thus the fine structure analysis furnishes us with constraining relations involving the weak and strong decay amplitudes of $D$ and $D^{\prime}$.

The presence of a dominant local doorway that gives rise to a large value of $\left|\gamma_{D s}^{(0)} / \gamma_{D p}^{(0)}\right|$ is certainly possible in some nuclei. The probability that $\left|\gamma_{D s}^{(0)} / \gamma_{D p}^{(0)}\right|$ is, say, $f$ can be calculated as follows. Since the local doorways are statistical in nature, the $\gamma_{D s}^{(0)}$ and $\gamma_{D p}^{(0)}$ are Gaussian distributed with about the same width. We thus have

$$
\begin{aligned}
P\left(\left|\frac{\gamma_{D s}^{(0)}}{\gamma_{D p}^{(0)}}\right|=f\right) & =\frac{1}{\pi} \int_{-\infty}^{\infty} d x \int_{-\infty}^{\infty} d y e^{-\left(x^{2}+y^{2}\right)} \delta\left(\left|\frac{x}{y}\right|-f\right) \\
& =\frac{2}{\pi} \frac{1}{1+f^{2}} .
\end{aligned}
$$

Thus, within our model, the probability for the occurence of the phenomenon of large parity violation with sign correlation goes as $\left(1+f^{2}\right)^{-1}$, which is small for $f \sim 4$ which we found in $\mathrm{n}+{ }^{232} \mathrm{Th}$. Therefore such a phenomenon is not a global one that is exhibited by nuclei over the periodic table. It happens in ${ }^{232} \mathrm{Th}$ due to a statistical fluctuation among the properties of local doorways. In fact, in the $\mathrm{n}+{ }^{238} \mathrm{U}$ system, also studied by the TRIPLE Group (Zhu et al,[1]), the average value of $P$ was found to be zero and its magnitude smaller so that $f \sim 1$. This implies that a local dominant $2 p-1 h$ doorway state, so conspicuous in ${ }^{233} \mathrm{Th}$, does not occur in ${ }^{239} \mathrm{U}$. With no single dominant doorway present, e.g. if two closely spaced doorways are relevant in the energy region of interest, the sign correlation disappears.

Our analysis points to the conclusion that the phenomenon of "sign correlation" is purely a conventional nuclear structure problem, and it is not connected to "exotics". Further, the phenomenon occurs in Th by statistical accident. Before ending we mention that in Th the single particle p-wave is resonant whereas the s-wave is not. Even so, $f \simeq\left|\gamma_{D s}^{(0)} / \gamma_{D p}^{(0)}\right|$ was found to be about 4. It seems to us that a more favorable case would be to have the pwave off-resonance and the s-wave on $(A \sim 170)$. This would give rise to a larger $f$, with higher probability. Of course, this will be experimentally more difficult because the $p$-wave resonances in this region may be too narrow. 


\section{ACKNOWLEDGEMENT}

We thank J. D. Bowman and H. Feshbach for useful discussions.

\section{REFERENCES}

[1] C. M. Frankle et al. Phys. Rev. Lett. 67, 564 (1991); C. M. Frankle et al. Phys. Rev. C46, 778 (1992). X. Zhu et al. Phys. Rev.C46, 768(1992); J. D. Bowman et al. Phys. Rev.48, 1116(1993); For a more recent review see J. D. Bowman et. al., Annu. Rev. Nucl. Part. Sci.43, 829 (1993).

[2] J. D. Bowman, G. T. Garvey, C. R. Gould, A. C. Hayes and M. B. Johnson, Phys. Rev. Lett.68, 780 (1992).

[3] N. Auerbach, Phys. Rev.C45, R514 (1992).

[4] N. Auerbach and J. D. Bowman, Phys. Rev.C46, 2582 (1992).

[5] C. H. Lewenkopf and H. A. Weidenmüller, Phys. Rev.C46, 2601 (1992).

[6] J. J. Szymanski, J. D. Bowman, M. Leuschner, A. B. Brown and I. C. Girit, Phys. Rev.C49, 3297 (1994).

[7] Fine structure analysis of processes involving discrete symmetry breaking such as iso-spin were made by A. F. R. de Toledo Piza and A. K. Kerman, Ann. Phys.48, 173 (1968)

[8] See, e.g., B. Block and H. Feshbach, Ann. Phys. 23, 47 (1963); A. K. Kerman, L. S. Rodberg and J. E. Young, Phys. Rev. Lett.11, 422 (1963); H. Feshbach, A. K. Kerman and R. M. Lemmer, Ann. Phys. 41, 280 (1967).

[9] H. Feshbach, "Theoretical Nuclear Physics: Nuclear Reactions" (John Wiley and Sons, New York, 1992)

[10] The exact size of these matrix elements needs further detailed investigation using estimates of the two-body PNC force. For a recent discussion see, e.g., A. C. Hayes and I. S. Towner, Phys. Lett.B302, 157 (1993). See also[4] 